УДК 537.874 .7

DOI: $10.17308 / \mathrm{kcmf} .2019 .21 / 712$

Поступила в редакцию 31.01 .2018

Подписана в печать 15.02.2019

\title{
ИСПОЛЬЗОВАНИЕ МИКРОВОЛНОВОЙ СПЕКТРОСКОПИИ ДЛЯ ИЗУЧЕНИЯ СОСТОЯНИЯ ПЕРЕОХЛАЖДЕННОЙ ВОДЫ
}

\author{
(C) 2019 Г. С. Бордонский*, А. А. Гурулев, С. Д. Крылов, С. В. Цыренжапов \\ Институт природных ресурсов, экологии и криологии СО РАН \\ а/я 1032, Недорезова, 16-а, 672002 Чита, Российская Федераџия
}

\begin{abstract}
Аннотация. Представлены методики экспериментов для изучения переохлажденной воды с использованием микроволновой спектроскопии. Одна методика связана с получением глубокого переохлаждения воды в порах силикатного материала, другая основана на получении аморфного состояния в образце пресного льда при его пластической деформации. Показаны возможности методик при изучении свойств переохлажденной воды. При атмосферном давлении и температуре $-45^{\circ} \mathrm{C}$ (на линии Видома) был определен интервал температур, в котором наблюдаются аномалии микроволновых потерь переохлажденной воды, находящейся в порах силикагеля. При пластической деформации поликристаллического льда наблюдали минимум фактора потерь в микроволновом диапазоне на линии Видома.
\end{abstract}

Ключевые слова: микроволновая спектроскопия, переохлажденная вода, линия Видома, структурные превращения.

\section{ВВЕДЕНИЕ}

Как известно, вода обладает многими аномалиями физико-химических свойств как при положительных, так и отрицательных температурах [1]. Одной из ярких особенностей глубоко переохлажденной метастабильной воды является ее вторая критическая точка перехода жидкость-жидкость, обнаруженная при компьютерном моделировании при температуре $-53{ }^{\circ} \mathrm{C}$ и давлении около $100 \mathrm{MПа}$ [2]. Влияние второй критической точки простирается в область низких давлений через особую линию на фазовой диаграмме температура-давление: линию Видома [3, 4]. Вблизи атмосферного давления температура на этой линии составляет $-45^{\circ} \mathrm{C}$. Предполагается, что многие аномалии холодной воды связаны со второй критической точкой.

Однако изучение переохлажденной воды затруднено из-за сложностей ее получения, так как жидкость находится в метастабильном состоянии. Особые сложности возникают при охлаждении воды ниже $-42^{\circ} \mathrm{C}$, температуры гомогенной нуклеации [4]. Последнее достижение - охлаждение капель микронных размеров в вакууме на время порядка миллисекунд до температуры $-46{ }^{\circ} \mathrm{C}$ [5]. Изучение структуры капель осуществляли с использованием импульсного рентгеновского лазера фемтосекундной длительности.

Бордонский Георгий Степанович, e-mail: lgc255@mail.ru
Отмеченные сложности можно в значительной степени преодолеть при охлаждении воды, находящейся в порах твердых тел. Однако исследование такой воды сложно осуществить оптическими и иными методами из-за влияния на излучение материала матрицы. Для поиска простого и эффективного способа изучения переохлажденной воды в работе [6, 7] была предложена методика микроволновой спектроскопии пористой увлажненной среды, основанная на измерении затухания излучения. Для устранения влияния матрицы на поглощение была выбрана среда с малым погонным затуханием по сравнению с водой. Такой средой оказались нанопористые силикатные материалы, широко используемые в качестве сорбентов (силикагели, специальные материалы $S B A-15, S B A-16, M C M-41$ и другие). Особо важно то, что большая часть объема поровой воды в этих материалах имеет свойства, близкие к свойствам объемной метастабильной воды [8-11]. Как было показано с использованием методов компьютерного моделирования кластеров воды в порах силикатов, преимущественно первый слой воды на поверхности пор оказывается прочно связанным с поверхностью материала. Последующие слои воды имеют параметры (плотность, число водородных связей на молекулу воды и другие), близкие к параметрам объемной воды. В то же время вода в порах достаточно стабильна, 
по крайней мере, на время, необходимое для проведения измерения ее характеристик. Связанная вода в порах твердых гидрофильных сред находится в равновесном состоянии из-за влияния энергии поверхности раздела, при этом близкие к поверхности слои имеют измененную структуру. Число таких слоев определяется химическими особенностями материала поверхности пор.

Другой особенностью объемной воды является ее точка фазового перехода лед-вода, равная при давлении 1 bar $0{ }^{\circ} \mathrm{C}$. Свойства воды при $0{ }^{\circ} \mathrm{C}$ трудно исследовать из-за неустойчивости ее фазового состояния при этой температуре и неоднозначности измеряемых характеристик при процессах охлаждения и нагревании, определяемых тепловой историей образцов. Для исследования такого состояния в [12] была использована методика измерения затухания при радиопросвечивания блоков льда, подвергающихся медленному нагреванию.

Цель настоящей работы - рассмотреть усовершенствованные методики микроволновой спектроскопии, которые можно использовать для более информативных исследований переохлажденной воды в широком интервале температур, например, для изучения химических превращений с ее участием в порах твердой фазы.

\section{МЕТОДИКИ МИКРОВОЛНОВОЙ СПЕКТРОСКОПИИ МИКРОВОЛНОВЫХ ИЗМЕРЕНИЙ}

Усовершенствование методик микроволновых измерений было направлено на получение более полной информации о состоянии переохлаждения воды в нанопористых средах, так и в монолитных ледяных образцах, подвергаемых пластической деформации. Возможность глубокого переохлаждения воды, близкой по свойствам к объемной метастабильной воде при использовании нанопористых силикатов, обсуждена выше. Недостаток этого способа - трудность его осуществления для температур ниже $-50 \ldots-70{ }^{\circ} \mathrm{C}$.

Вместе с тем, при исследованиях льда вблизи $0{ }^{\circ} \mathrm{C}$ [12] был сделан вывод о том, что при нагревании блока льда в нем возникают температурные напряжения и возникает пластическая деформация при скольжении частей кристаллов льда $I h$ по базисным плоскостям. Тот же эффект имеет место, если лед образуется из воды в пространстве, ограниченном прочными стенками $[13,14]$. В этом случае в среде образуются слои с разорванными водородными связями, которые могут быть отождествлены с аморфной фракцией или жидкос- тью. В принципе, таким способом можно получать аморфное состояние, близкое к жидкому при любых температурах и давлениях. Пластическую деформацию можно осуществить при механическом воздействии на образец льда, однако использование температурных напряжений в твердой фазе является более удобным способом, так как позволяет сохранять монолитность образцов. Некоторые отличия свойств образующейся фазы с разорванными водородными связями от жидкости в этом случае, по-видимому, будут связаны с ее взаимодействием с кристаллическими слоями. Этот вопрос, о соответствии текучей фазы свойствам объемной воде, требует специального изучения. Однако эта методика представляет большой интерес, так как позволяет достичь температур переохлаждения в области «no man's land»: $-37 \ldots-120^{\circ} \mathrm{C}[1,4]$.

Другой путь совершенствования методик измерений относится к инструментальным способами обработки зондирующих сигналов. Для этого необходимо: 1 - расширить спектральный диапазон от единиц гигагерц до частот терагерцового диапазона $\sim 200 \mathrm{GHz}$ (так как погонное затухание существенно изменяется с частотой); 2 - измерять не только интенсивность прошедшего излучения, но и сдвиг фазы волны $(\varphi)$, а также комплексный коэффициент отражения, то есть его модуль и фазу; 3 - выбирать определенные режимы нагревания и охлаждения образцов для выявления нелинейных свойств среды. Схемы измерительных установок, реализующие отмеченные усовершенствования, представлены на рис. $1 a, b$.

В схеме на рис. $1 a$ измеряется пропускание мощности излучения через увлажненную нанопористую среду или лед в зависимости от температуры и ее скорости изменения. Образец (сыпучий или твердый) размещается в волноводной секции. Генератор (1) и приемник (5) могут иметь частоты от 5 до $200 \mathrm{GHz}$. Программируемая климатическая камера (2) имеет диапазон регулировки температуры от +150 до $-65^{\circ} \mathrm{C}$ (авторы использовали камеру Espec $S U-261)$. При циклическом изменении температуры появляется возможность изучения гистерезисных явлений, связанных с фазовыми превращениями воды в пористых средах, а также компоненты во льду, обладающей текучестью. По схеме рис. $1 b$ выполняются измерения твердых пористых и монолитных образцов. В этой схеме образец имеет форму усеченной неравнобедренной призмы для устранения интерференции сигнала, излучаемого векторным анализатором, при отражении от противоположных граней. В данной методике кроме мо- 


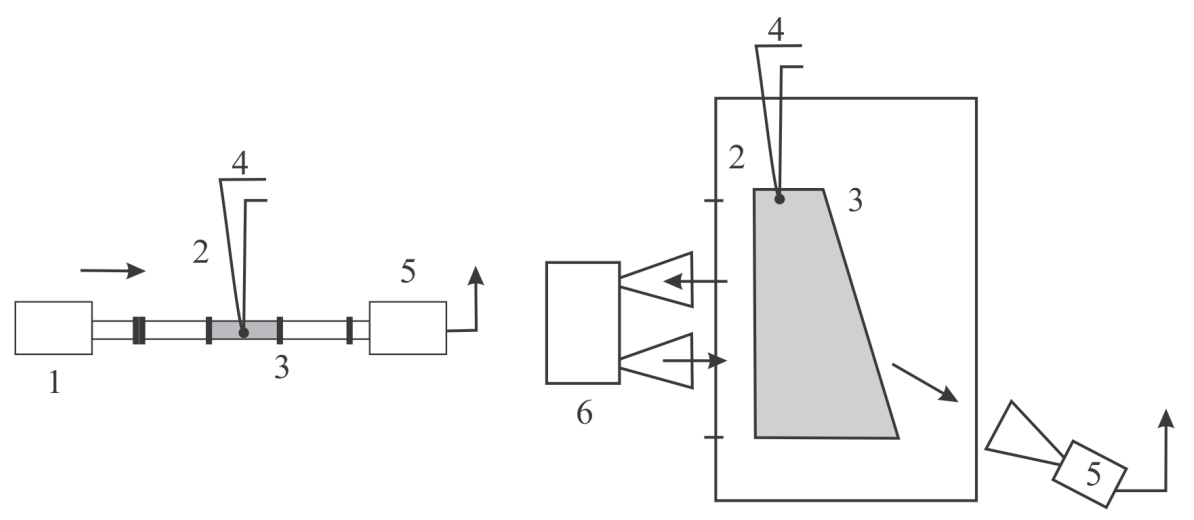

a)

b)

Рис. 1. Схемы установок для микроволновой спектроскопии переохлажденной воды в дисперсных средах и монолитных образцах льда, подвергающихся пластической деформации: $a$ ) измерения поглощения; $b$ ) измерения параметров отраженных и проходящих сигналов. 1 - генератор микроволнового излучения; 2 - программируемая климатическая камера; 3 - образец; 4 - термопара для измерения температуры образца; 5 - приемник излучения; 6 - векторный анализатор сигналов

[Fig. 1. Schematics of experimental setup to microwave spectroscopy of the supercooled water in the dispersed media and monolithic samples of ice which are under go to a plastic deformation: $a$ ) absorption measurement; $b$ ) measurement of parameters of the reflected and propagated signals. 1 - microwave generator; 2 - programmable climatic camera; 3 -sample;

4 - thermocouple for measurement of sample temperature; 5 - radiation receiver; 6 - vector signal analyzer]

дуля амплитуды отраженного и прошедшего через образец зондирующего излучения определяется и сдвиг фаз между падающей и отраженной волнами. Измерения температуры образцов выполняются с помощью термопары (4).

\section{НЕКОТОРЫЕ РЕЗУЛЫТАТЫ ИЗМЕРЕНИЙ}

С использованием представленных методик измерений были выполнены исследования состояния воды в силикагеле вблизи $-45^{\circ} \mathrm{C}$, а также ее поведения в деформируемых ледяных структурах при различных температурах. Ранее в [15] установлена возможность ускорения химических превращений при $-45^{\circ} \mathrm{C}$. Химические реакции могут ускоряться при данной температуре из-за резкого возрастания флуктуаций энтропии $(S)$ и плотности $(\rho)$ на линии Видома $[3,4]$. Согласно [16], средний квадрат флуктуаций энтропии пропорционален $C_{p}$ - теплоемкости при постоянном давлении по формуле $\left\langle(\Delta S)^{2}\right\rangle=k C_{p}$, где $k$ - постоянная Больцмана. Резкое возрастание $C_{p}$ при приближении к $-45^{\circ} \mathrm{C}$ установлено в ряде работ [2-4]. Поэтому эффект ускорения химических реакций может объясняться тем, что флуктуации энтропии связаны с флуктуациями энергии, которые позволяют с большей вероятностью преодолеть энергетический барьер реакций.

В настоящем исследовании авторы ставили задачу определения температурного интервала существенного возрастания интенсивности флуктуации энергии. Этот вопрос интересен в связи с нахождением условий ускорения химических реакций с участием жидкой переохлажденной воды. Возрастание флуктуаций энергии дипольной жидкости должно отражаться на поглощении электромагнитного излучения из-за роста восприимчивости среды при разрыве водородных связей. Была использована схема рис. $1 a$, по которой измеряли мощность прошедшего излучения через увлаженный порошкообразный силикагель марки КСКГ (со средними размерами пор $8 \mathrm{~nm}$ ) на частоте $20 \mathrm{GHz}$ в зависимости от температуры. Регистрацию сигналов осуществляли при помощи системы сбора информации фирмы «Agilent». Время опроса этой системой выходных сигналов приемника и напряжения термопары составляло $1 \mathrm{~s}$. Точность измерения температуры около $1^{\circ} \mathrm{C}$. Результаты одного из опытов приведены на рис. $2 a$. Как следует из полученных данных, при температуре вблизи $-45^{\circ} \mathrm{C}$ наблюдали резкое поглощение излучения. В различных экспериментах температура этого эффекта изменялась в пределах нескольких градусов, а ширина области поглощения составляла около 0.1 С. Эти данные совпадают с ранее полученными результатами в работе [15]. На рис. $2 b$ приведен график регистрации мощности, проходящего через образец сигнала, при возникновении реакции хемадсорбции водорода на поверхность пор силикагеля при температуре $-45^{\circ} \mathrm{C}$. Так же наблюдали импульс тепловыделения. Скорость изменения температуры образца силикагеля в экспериментах [15] на часто- 
те $34 \mathrm{GHz}$ составляла $\sim 2 \mathrm{deg} / \mathrm{min}$.

Анализ полученных данных показывает, что наиболее интенсивные вариации микроволновых сигналов наблюдались в интервале температур не более одного градуса Цельсия. Следует отметить, что влияние линии Видома на тепловые величины (теплоемкости при постоянном давлении, изотермической сжимаемости, коэффициента теплового расширения) [2-4], а также фактора потерь переохлажденной воды для микроволнового излучения [7], простирается на интервал порядка десяти градусов. Однако эти параметры непосредственно при температуре $-45^{\circ} \mathrm{C}$ для переохлажденной воды никем не измерялись. Исключение составляет работа [18], где исследование было выполнено для воды в порах силикагеля с их размерами $1.7 \ldots 2.4 \mathrm{~nm}$. Для среды с такими порами резкого изменения тепловых величин не наблюдали, их возрастание составило значения порядка десятков процентов при изменении температуры в интервале $+/-10{ }^{\circ} \mathrm{C}$.

Исследование деформируемого льда по схеме рис. $1 a$ проводили на образцах в волноводе, полученных замораживанием сверхчистой воды. Вода получена на установке Direct-Q3, ее электропроводность при комнатной температуре составила $0.05 \mu \mathrm{S} / \mathrm{cm}$. Для плавности процесса использовали методику создания во льду термических напряжений, определяемых градиентом температуры. Он возникает в образцах при их нагревании или охлаждении. При скоростях нагрева $1 \ldots 10^{\circ} \mathrm{C} / \min$ в образцах не наблюдали трещин и разрушения. Предполагалось, что пластическая деформация происходила, в основном, путем сдвига вдоль базисных плоскостей гексагональных кристаллов льда.

В эксперименте с исследованием аморфной (текучей) компоненты льда, которая, как предполагается, в определенной степени обладает свойствами жидкой воды, измерения выполнены на частоте $125 \mathrm{GHz}$. В качестве приемника излучения применен модуляционный радиометр; в качестве генератора использовали умножитель частоты на полупроводниковом диоде (частота задающего генератора $25 \mathrm{GHz}$ ). Результаты измерений приведены на рис. 3. Наблюдали зависимость проходящей мощности от температуры при ее линейном возрастании от -62 до $-10{ }^{\circ} \mathrm{C}$. Проходящая мощность достигла максимума при $-45^{\circ} \mathrm{C}$. Точность измерения температуры составляла $1{ }^{\circ} \mathrm{C}$.

Совпадение минимума потерь при $-45{ }^{\circ} \mathrm{C}$ (рис. 3) со значением температуры на линии Ви-

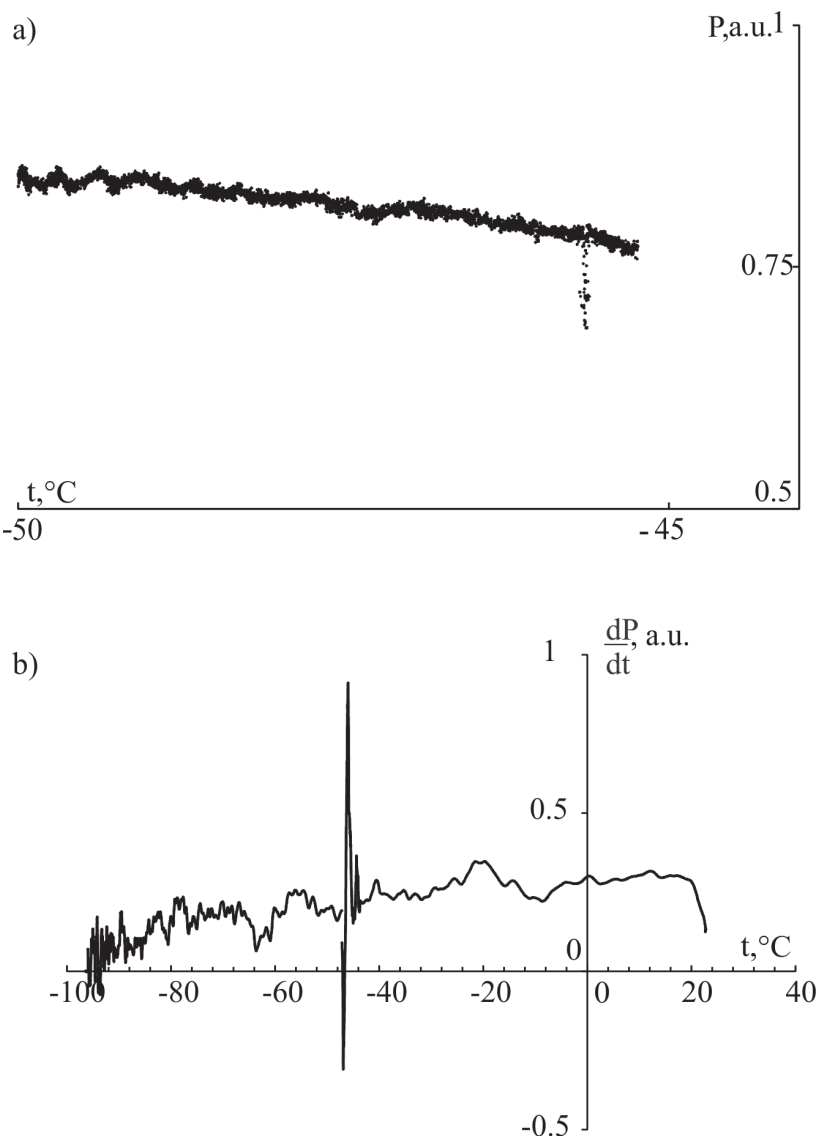

Рис. 2. Результаты измерений: $a$ ) мощности излучения (Р) на частоте $20 \mathrm{GHz}$, прошедшего через образец увлажненного силикагеля, находящегося в волноводной ячейке, с весовой влажностью 20 \% в процессе охлаждения; $b$ ) производной мощности прошедшего излучения на частоте $34 \mathrm{GHz}$ в зависимости от температуры при охлаждении от $20{ }^{\circ} \mathrm{C}$. Мощность представлена в относительных единицах

[Fig. 2. Observed datas: $a$ ) power of the radiation (P) at a frequency of $20 \mathrm{GHz}$ which propogate through example of the wet silica gel which is in a waveguide cell with moisture content of $20 \%$ in the course of cooling; $b$ ) power derivation of propagated radiation at $34 \mathrm{GHz}$ depending on temperature when cooling from $20{ }^{\circ} \mathrm{C}$. Power is represented at arbitrary units]

дома представляется не случайным. Отсюда следует, что текучая компонента в образце обладает свойствами жидкой переохлажденной метастабильной воды. Однако такая вода, как оказалось, имеет более низкий фактор потерь, чем кристаллический лед. Возможное объяснение связано с тем, что образующаяся вода имеет вид тонких пленок, то есть двумерных структур. Их высокочастотная проводимость существенно отлична ото льда и объемной воды и имеет меньшее значение.

Аналогичный эксперимент для блока льда в свободном пространстве проведен по схеме рис. $1 b$. 
Образец изымали из ледяного покрова пресного озера, его линейные размеры составляли значение порядка десятков сантиметров. Измерения выполнены на частотах 9..13.5 GHz. Блок льда переносили в лабораторное помещение, находящееся при температуре $+20^{\circ} \mathrm{C}$, где он нагревался от исходной температуры -10 до $0^{\circ} \mathrm{C}$.

Для получения информации о структуре льда измеряли комплексный коэффициент отражения, конкретно - мощность отраженного излучения и его фазу. Мощность отраженной волны определяется значением действительной части диэлектри- ческой проницаемости образца и, следовательно, появлением в нем жидкости, а также ее распределением в объеме исследуемого тела. Фаза коэффициента отражения характеризует как значения действительной и мнимой частей диэлектрической проницаемости, так и особенности структуры, например, появлением слоев с различными электромагнитными свойствами.

В выполненном эксперименте были обнаружены флуктуации фазы коэффициента отражения, которые имели выраженную частотную зависимость - рис. 4. Их экстремум соответствовал час-

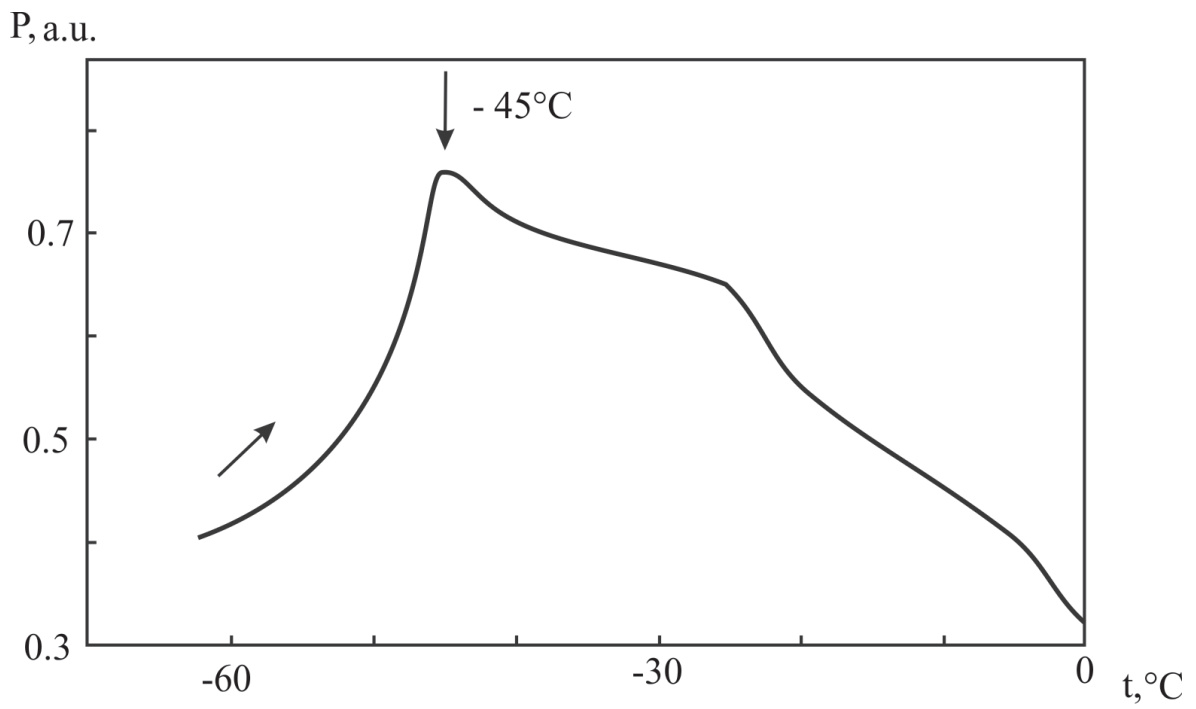

Рис. 3. Зависимость проходящей через лед мощности излучения на частоте $125 \mathrm{GHz}$ от температуры. Мощность представлена в относительных единицах

[Fig. 3. Temperature dependence of the radiation propagating through ice at a frequency of $125 \mathrm{GHz}$. Power is represented at arbitrary units]

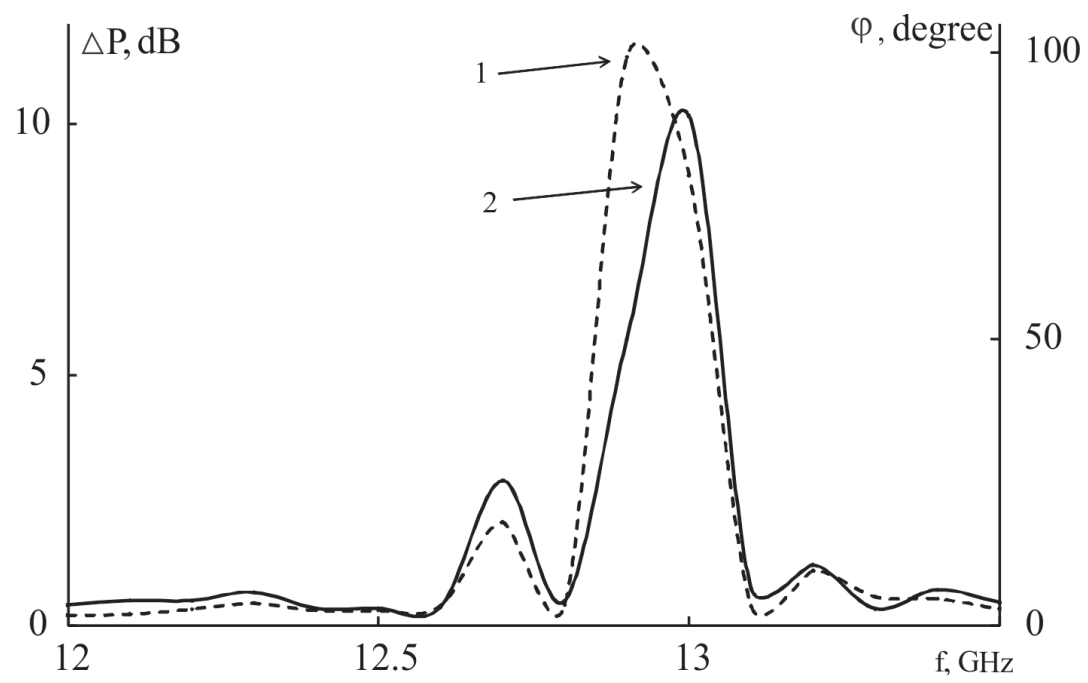

Рис. 4. Зависимости амплитуды флуктуаций фазы (1) и приращений мощности отраженного излучения (2) от частоты для блока пресного льда при температуре $-5^{\circ} \mathrm{C}$

[Fig. 4. Frequency dependences of phase amplitude of fluctuations (1) and increments of the reflected radiation power (2) for the block of fresh ice at a temperature of $\left.-5^{\circ} \mathrm{C}\right]$ 
тоте $13 \mathrm{GHz}$ (длина волны $1.35 \mathrm{~cm}$ во льду). Полученный результат можно связать с возникновением во льду волн течения сантиметровой длины при пластической деформации [19]. Эти волны, имеющие вид дифракционных решеток, хаотически распределены в объеме образца, что и приводит к флуктуациям $\varphi$.

\section{ВЫВОДЫ}

Предложенные методики микроволнового исследования переохлажденной воды в поровом пространстве нанопористых силикатов и льда, подвергающегося пластической деформации, дополняют известные методы ее изучения. В данной статье были продемонстрированы возможности усовершенствованных методик и показано следующее.

1. Определен температурный интервал наиболее сильных флуктуаций энтропии и плотности воды вблизи $-45^{\circ} \mathrm{C}$ на линии Видома при давлении $0.1 \mathrm{MPa}$, который составляет по данным микроволновой спектроскопии значение $0.1 \ldots 1{ }^{\circ} \mathrm{C}$.

2. Обнаружен минимум электромагнитных потерь при $-45^{\circ} \mathrm{C}$ на частоте $125 \mathrm{GHz}$, который показывает существование особенности при этой температуре. Совпадение температур минимума потерь и теоретического значения на линии Видома подтверждает ее существование.

3. Методика измерений с использованием векторного анализатора излучения, с помощью которого обнаружено возрастание флуктуаций фазы отраженного от блока льда монохроматического излучения на частоте $13 \mathrm{GHz}$, позволила подтвердить существование волн макроскопической пластичности сантиметровой длины в поликристаллической среде.

\section{ИСТОЧНИК ФИНАНСИРОВАНИЯ}

Работа выполнена при финансовой поддержке РФФИ, проект № 18-05-00085.

\section{КОНФЛИКТ ИНТЕРЕСОВ}

Авторы декларируют отсутствие явных и потенциальных конфликтов интересов, связанных с публикацией настоящей статьи.

\section{СПИСОК ЛИТЕРАТУРЫ}

1. Chaplin M. Water Structure and Science. URL: http://www.lsbu.ac.uk/water/chaplin.html (дата обращения: 18.01.2019).

2. Mishima O. // Journal of Chemical Physics, 2010, vol. 133, № 14, p. 144503/6. DOI: 10.1063/1.3487999
3. Xu L., Kumar P., Buldyrev S. V., Chen S.-H., Poole P. H., Sciortino F., Stanley H. E. // Proceedings of the National Academy of Sciences of the United States of America, 2005, vol. 102, iss. 46, pp. 16558-16562. DOI: 10.1073/pnas.0507870102

4. Franzese G., Stanley H. E. // Journal of Physics Condensed Matter, 2007, vol. 19, p. 205126/1-16. DOI: 10.1088/0953-8984/19/20/205126

5. Sellberg J. A., Huang C., McQueen T. A., Loh N. D., Laksmono H., Schlesinger D., Sierra R. G., Nordlund D., Hampton C. Y., Starodub D., Deponte D. P., Beye M., Chen C., Martin A. V., Barty A., Wikfeldt K. T., Weiss T. M., Caronna C., Feldkamp J., Skinner L. B., Seibert M. M., Messerschmidt M., Williams G. J., Boutet S., Pettersson L. G. M., Bogan M. J., Nilsson A. // Nature, 2014, vol. 510, no. 7505 , pp. 381-384. DOI: $10.1038 /$ nature 13266

6. Бордонский Г. С., Крылов С. Д. // Журнал физической химии, 2012, т. 86, № 11, с. 1806.

7. Бордонский Г. С., Гурулев А. А., Крылов С. Д., Сигачев Н. П., Щегрина К. А. // Конденсированные среды и межфазные гранищы, 2016, т. 18, № 3, с. 304311.

8. Castrillyn S. R.-V., Giovambattista N., Aksay U. A., Debenedetti P. G. // Journal of Physical Chemistry B, 2009, vol. 113, iss. 23, pp. 7973-7976. DOI: 10.1021/jp9025392

9. Cerveny S., Mallamace F., Swenson J., Vogel M., Xu L. // Chemical Reviews, 2016, vol. 116, iss. 13, pp. 76087625. DOI: $10.1021 /$ acs.chemrev.5b00609

10. Gallo P., Rovere M., Chen S.-H. // Journal of Physical Chemistry Letters, 2010, vol. 1, iss. 4, pp. 729-733. DOI: $10.1021 / \mathrm{jz} 9003125$

11. Меньшиков Л. И., Меньшиков П. Л., Федичев П. О. // Журнал экспериментальной и теоретической физики, 2017, т. 152, № 6, с. 1374-1392.

12. Бордонский Г. С., Гурулев А. А., Крылов С. Д. // Радиотехника и электроника, 2014, т. 59, № 6, с. 587592.

13. Бордонский Г. С., Крылов С. Д. // Письма в ЖТФ, 2017, т. 43, № 21, c. 64-71.

14. Silonov V. M., Chubarov V. V. // Journal of Surface Investigation, 2016, vol. 10 , iss. 4, pp. 883-886. DOI: $10.1134 / \mathrm{S} 1027451016030356$

15. Бордонский Г. С., Гурулев А. А. // Письма в ЖТФ, 2017, т. 43, № 8, с. 34-40.

16. Ландау Л. Д., Лифшиц Е. М. Теоретическая физика. Том. 5. Статистическая физика. Часть 1. М.: Физматлит, 2002, 616 c.

17. Орлов А. О. // Вестник Забайкальского государственного университета, 2016, т. 22, № 8, с. 14-20.

18. Nagoe A., Kanke Y., Oguni M., Namba S. // Journal of Physical Chemistry B, 2010, vol. 114, iss. 44, pp. 1394013943. DOI: $10.1021 / \mathrm{jp} 104970$ s

19. Зуев Л. Б. // Успехи физики металлов, 2015, т. 16, № 1, c. 35-60. 
UDC 537.874.7

https://doi.org:

DOI: $10.17308 / \mathrm{kcmf} .2019 .21 / 712$

Received 31.01.2019

Accepted 15.02.2019

\title{
USING MICROWAVE SPECTROSCOPY TO STUDY THE STATE OF SUPERCOOLED WATER
}

\author{
(C) 2019 G. S. Bordonskiy*, A. A. Gurulev, S. D. Krylov, S. V. Tsyrenzhapov \\ Institute of Natural Resources, Ecology and Cryology, Siberian Branch of the Russian Academy of Sciences \\ POB 1032, 16-a Nedorezova str., 672002 Chita, Russian Federation
}

\begin{abstract}
Objective. One of the anomalies of water is its second critical point of the liquid-liquid transition at a temperature of $-53^{\circ} \mathrm{C}$ and a pressure of about $100 \mathrm{MPa}$. It is known that on the pressure-temperature diagram the so-called Widom line flows from this point into a single-phase region. This line is characteristic of increased entropy fluctuations and water density. At a pressure of $0.1 \mathrm{MPa}$, the temperature on the Widom line is $-45^{\circ} \mathrm{C}$. This temperature is reached in Earth's polar regions and atmosphere. It is, therefore, important to investigate the physical and chemical processes determined by the second critical point of water. However, the study of deeply supercooled water is difficult due to the lack of a technology for its production. For this reason, the temperature range from -37 to $-120^{\circ} \mathrm{C}$ is called "no man's land". This complexity can be overcome by cooling water in the pores of solid bodies. It is also possible to produce supercooled water by creating an amorphous phase in ice.

Methods and methodology. This paper presents methods for the study of supercooled water in the pores of silicate materials and in the case of ice amorphization. Amorphization was achieved with plastic deformation caused by a temperature gradient. The techniques are based on the measurements of water microwave characteristics in samples since silicates and polycrystalline ice are sufficiently transparent for microwave radiation and do not have a significant effect on it. The distinctive features of the techniques are associated with the expansion of the range of used frequencies from 5 to $200 \mathrm{GHz}$ and the measurement of the intensity and the phase of the transmitted and the reflected radiation. In case of amorphization, the peculiarities are associated with the creation of special heating and cooling modes for ice samples.

Results. As an example, the study presents the results of determining the temperature range on the Widom line, for which increased entropy fluctuations and density of supercooled water were observed. This range was about $1{ }^{\circ} \mathrm{C}$. During the plastic deformation of ice caused by the heating of the sample, a decrease in microwave losses was found in the proximity of $-45^{\circ} \mathrm{C}$. Strong fluctuations in the phase of radiation reflected from a block of fresh polycrystalline ice with an extremum in proximity of $13 \mathrm{GHz}$ were also detected.

Conclusions. This effect is supposed to be associated with the emergence of macrolocalized plasticity waves. Thus, the proposed methods of microwave spectroscopy of supercooled water can complement the known methods used to study its state.
\end{abstract}

Keywords: microwave spectroscopy, supercooled water, Widom line, structural transformations.

\section{SOURCE OF FINANCING}

The study was supported by the Russian Foundation for Basic Research (grant No. 18-05-00085).

\section{CONFLICT OF INTEREST}

The authors declare the absence of obvious and potential conflicts of interest related to the publication of this article

$\checkmark$ Bordonskiy Georgy S., e-mail: lgc255@mail.ru

\section{REFERENCES}

1. Chaplin M. Water Structure and Science. URL: http://www.lsbu.ac.uk/water/chaplin.html (accessed 18 January 2019).

2. Mishima O. Journal of Chemical Physics, 2010, vol. 133, no. 14, p. 144503/6. DOI: 10.1063/1.3487999/

3. Xu L., Kumar P., Buldyrev S. V., Chen S.-H., Poole P. H., Sciortino F., Stanley H. E. Proceedings of the National Academy of Sciences of the United States of 
America, 2005, vol. 102, iss. 46, p. 16558-16562. DOI: 10.1073/pnas.0507870102

4. Franzese G., Stanley H. E. Journal of Physics Condensed Matter, 2007, vol. 19, p. 205126/1-16. DOI: 10.1088/0953-8984/19/20/205126

5. Sellberg J. A., Huang C., McQueen T. A., Loh N. D., Laksmono H., Schlesinger D., Sierra R. G., Nordlund D., Hampton C. Y., Starodub D., Deponte D. P., Beye M., Chen C., Martin A. V., Barty A., Wikfeldt K. T., Weiss T. M., Caronna C., Feldkamp J., Skinner L. B., Seibert M. M., Messerschmidt M., Williams G. J., Boutet S., Pettersson L. G. M., Bogan M. J., Nilsson A. Nature, 2014, vol. 510, no. 7505, pp. 381-384. DOI: $10.1038 /$ nature 13266

6. Bordonskiy G. S., Krylov S. D. Russian Journal of Physical Chemistry A, vol. 86, iss. 11, pp. 1682-1688. DOI: 10.1134/S0036024412110064

7. Bordonskiy G. S., Gurulev A. A., Krylov S. D., Sigachev N. P., Schegrina K. A. Condensed Matter and Interphases, 2016, vol. 18, no. 3, pp. 304-311. (in Russ.)

8. Castrillyn S. R.-V., Giovambattista N., Aksay U. A., Debenedetti P. G. Journal of Physical Chemistry B, 2009, vol. 113, iss. 23, pp. 7973-7976. DOI: 10.1021/jp9025392

9. Cerveny S., Mallamace F., Swenson J., Vogel M., Xu L. Chemical Reviews, 2016, vol. 116, iss. 13, pp. 76087625. DOI: 10.1021 acs.chemrev.5b00609

10. Gallo P., Rovere M., Chen S.-H. Journal of Physical Chemistry Letters, 2010, vol. 1, iss. 4, pp. 729-733. DOI: 10.1021/jz9003125
11. Menshikov L. I., Menshikov P. L., Fedichev P. O. Journal of Experimental and Theoretical Physics, vol. 125, iss. 6, pp. 1173-1188. DOI: 10.1134/S1063776117120056

12. Bordonskii G. S., Gurulev A. A., Krylov S. D. Journal of Communications Technology and Electronics, vol. 59, iss. 6, pp. 536-540. DOI: 10.1134/ S1064226914060060

13. Bordonskii G. S., Krylov S. D. Technical Physics Letters, vol. 43, iss. 11, pp. 983-986. DOI: 10.1134/ S1063785017110025

14. Silonov V. M., Chubarov V. V. Journal of Surface Investigation, 2016, vol. 10, iss. 4, pp. 883-886. DOI: $10.1134 / \mathrm{S} 1027451016030356$

15. Bordonskii G. S., Gurulev A. A. Technical Physics Letters, vol. 43, iss. 4, pp. 380-382. DOI: 10.1134/ S1063785017040174

16. Landau L. D., Lifshic E. M. Teoreticheskaya fizika. Tom. 5. Statisticheskaya fizika. CHast'1. M.: Fizmatlit Publ., 2002, 616 p. (in Russ.).

17. Orlov A. O. Vestnik Zabajkal'skogo gosudarstvennogo universiteta, 2016, vol. 22, no. 8, pp. 14-20. (in Russ.)

18. Nagoe A., Kanke Y., Oguni M., Namba S. Journal of Physical Chemistry B, 2010, vol. 114, iss. 44, pp. 1394013943. DOI: $10.1021 /$ jp $104970 \mathrm{~s}$

19. Zuev L. B. Usp. Fiz. Met., 2015, vol. 16, no. 1, pp. 35-60. (in Russ.).
Бордонский Георгий Степанович - д. ф.-м. н., профессор, зав. лабораторией геофизики криогенеза, Институт природных ресурсов, экологии и криологии Сибирского отделения Российской академии наук, Чита, Российская Федерация; e-mail: 1gc255@mail.ru. ORCID iD 0000-0002-0009-0822.

Гурулев Александр Александрович - к. ф.-м. н., c. н. с., доцент, Институт природных ресурсов, экологии и криологии Сибирского отделения Российской академии наук, Чита, Российская Федерация; e-mail: sansang@mail.ru. ORCID iD 00000003-2232-3583.

Крылов Сергей Дмитриевич - к. ф.-м. н., с. н. с., Институт природных ресурсов, экологии и криологии Сибирского отделения Российской академии наук, Чита, Российская Федерация; e-mail: krylov_ s_d@mail.ru. ORCID iD 0000-0003-2920-1722.

Цыренжапов Сергей Васильевич - м. н. с., Институт природных ресурсов, экологии и криологии Сибирского отделения Российской академии наук, Чита, Российская Федерация; e-mail: arahley@mail. ru. ORCID iD 0000-0002-7590-5619.
Bordonskiy Georgy S. - Dr. Sci. (Phys.-Math.), Professor, Head of the Laboratory of Geophysics of Cryogenesis, Institute of Natural Resources, Ecology and Cryology Siberian Branch of the Russian Academy of Sciences, Chita, Russian Federation; 1gc255@mail.ru. ORCID iD 0000-0002-0009-0822.

Gurulev Aleksandr A. - Cand. Sci. (Phys.-Math.), Senior Researcher, Associate Professor, Institute of Natural Resources, Ecology and Cryology Siberian Branch of the Russian Academy of Sciences, Chita, Russian Federation; e-mail: sansang@mail.ru. ORCID iD 0000-0003-2232-3583.

Krylov Sergey D. - Cand. Sci. (Phys.-Math.), Senior Researcher, Institute of Natural Resources, Ecology and Cryology Siberian Branch of the Russian Academy of Sciences, Chita, Russian Federation; e-mail: krylov_s_d@mail.ru. ORCID iD 0000-00032920-1722.

Tsyrenzhapov Sergey V. - Junior Researcher, Institute of Natural Resources, Ecology and Cryology Siberian Branch of the Russian Academy of Sciences, Chita, Russian Federation; e-mail: arahley@mail.ru. ORCID iD 0000-0002-7590-5619. 